\title{
Analysis and Application of A One-Layer Neural Network for Solving Horizontal Linear Complementarity Problems*
}

\author{
Xingbao Gao ${ }^{1}$ and Jing Wang ${ }^{2}$ \\ ${ }^{1}$ College of Mathematics and Information Science, \\ Shaanxi Normal University, \\ Xi'an, Shaanxi, 710062 China \\ E-mail:xinbaog@snnu.edu.cn \\ ${ }^{2}$ Institute of Information and System Science, \\ Beifang University of Nationalities, \\ Yinchuan, Ningxia, 750021 China \\ E-mail: jingwang0106@yahoo.com.cn
}

Received 31 May 2012

Accepted 29 March 2013

\begin{abstract}
In this paper, we analyze the stability and convergence of a one-layer neural network proposed by Gao and Wang, which is designed to solve a class of horizontal linear complementarity problems. The globally asymptotical stability and globally exponential stability of this network are proved strictly under mild conditions, respectively. Meanwhile, this network is applied to solve a transportation problem and a class of the absolute equations.
\end{abstract}

Keywords: Horizontal linear complementarity problem, neural network, stability, application

\section{Introduction}

Let $A, B \in R^{n \times n}$ and $q \in R^{n}$ be given, then the horizontal linear complementarity problem $(\operatorname{HLCP}(A, B, q))^{1,2}$ is to find the vectors $x$ and $y \in R^{n}$ such that

$$
A x-B y=q, \quad x \geqslant 0, \quad y \geqslant 0, \quad x^{T} y=0 .
$$

Obviously problem (1) includes the following linear complementarity problem LCP $(M, q)$ : find a vector $y \in R^{n}$ such that

$$
y \geqslant 0, \quad M y+q \geqslant 0, \quad y^{T}(M y+q)=0,
$$

where $M \in R^{n \times n}$ and $q \in R^{n}$. Then it is a fundamental problem in mathematical programming and provides a general framework to study linear and quadratic programming problems, bi-matrix games as well as more general equilibrium problems and variational inequality problems (see Refs. 1-7 and the references therein). Thus problem (1) has many important applications in scientific and engineering fields including network economics, transportation science, structure design, mechanical design, electrical engineering and so on (see Refs. 1-9 and the references therein). In many practical applications, problem (1) is desired to be solved in real time.

*This research was supported in part by grants from the National Natural Science Foundation of China (No. 61273311; 61173094), and Fundamental Research Funds for the Central Universities (GK201001002). 
However, traditional algorithms (see Refs. 7-13 and the references therein) may not be effective since the computing time required for a solution is greatly dependent on the dimension and the structure of the problem, and the complexity of the algorithm used. One promising approach to handle these problems with high dimension and dense structure is to employ artificial neural network based circuit implementation. Even though the existing neural-network models in Refs. 14-22 can be used to solve problem (1) by transforming it into a LCP in (2) when matrix $A$ or $B$ is nonsingular, their stability and convergence might not be guaranteed since the resulting LCP does not satisfy the stability conditions of these models (see Example 1 in Section 4 and Ref. 15). Moreover, matrices $A$ and $B$ are singular in practical problems (see Example 2 in Section 4 and Ref. 15), and the models in Refs. 16-19 are rather complex since they have two-layer structure. Recently, Gao and Wang ${ }^{15}$ proposed the following a one-layer neural network model for (1):

- state equation

$$
\frac{d z}{d t}=-\rho\left[x-(2 x-z)^{+}\right]
$$

- output equation

$$
\left\{\begin{array}{l}
x=R z+s \\
y=D(z-x)
\end{array}\right.
$$

where $\rho>0$ is a scaling constant, $x^{+}=\left(x_{1}^{+}, x_{2}^{+}\right.$, $\left.\cdots, x_{n}^{+}\right)^{T}$ with $x_{i}^{+}=\max \left\{x_{i}, 0\right\}$ for $i=1,2, \cdots, n$, $R=W^{-1} B D, s=W^{-1} q, W=A+B D$ is nonsingular and $D=\operatorname{diag}\left(d_{1}, d_{2}, \cdots, d_{n}\right)$ is a positive diagonal matrix $\left(d_{i}>0\right.$ for $\left.i=1,2, \cdots, n\right)$. This model has a low complexity and can be implemented by using simple hardware units. Even though Gao and Wang ${ }^{15}$ pointed out that (3)-(4) is asymptotically stable and globally exponentially stable under the mild condition respectively, no proof and application are given. Since this model can solve some nonmonotone problems (see Example 1 in Section 4 and Examples 1-3 in Ref. 15) and the stability analysis can build the foundation for its application, it is necessary to prove strictly the stability results of (3)-(4) in Ref. 15 and provide some applications.
Besides the above consideration, motivated by the importance of the stability, convergence and exponential stability of equilibrium points for neural networks in many continuous optimization problems(see Refs.20-22 and the references therein), we analyze the stability and convergence of neural network (3)-(4) in this paper. The globally asymptotical stability and globally exponential stability of (3)(4) are strictly proved under mild conditions, respectively. Meanwhile, it is applied to solve a transportation problem and a class of the absolute equations.

Throughout the paper, we assume that the solution set $\mathscr{S}^{*}=\left\{(x, y) \in R^{2 n} \mid(x, y)\right.$ solves $\left.(1)\right\} \neq \emptyset$, and that there exists a positive diagonal matrix $D=$ $\operatorname{diag}\left(d_{1}, d_{2}, \cdots, d_{n}\right)\left(d_{i}>0\right.$ for $\left.i=1,2, \cdots, n\right)$ such that the matrix $W=A+B D$ is nonsingular.

In our following discussions, we let $\|\cdot\|$ denote the Euclidean norm, $I_{n}$ denote the identity matrix of order $n, R_{+}^{n}=\left\{x \in R^{n} \mid x_{i} \geqslant 0\right.$ for $i=$ $1,2, \cdots, n\}, \nabla \varphi(x)=\left(\partial \varphi(x) / \partial x_{1}, \partial \varphi(x) / \partial x_{2}, \cdots\right.$, $\left.\partial \varphi(x) / \partial x_{n}\right)^{T} \in R^{n}$ denote the gradient vector of the differentiable function $\varphi(x)$ at $x$. For any vector $u \in R^{n}, u^{T}$ denotes its transpose. For any $n \times n$ real symmetric matrix $M, \lambda_{\min }(M)$ and $\lambda_{\max }(M)$ denote its minimum and maximum eigenvalues, respectively. A neural network is said to be stable in the sense of Lyapunov, globally asymptotically stable, if the corresponding dynamical system is so.

The rest of the paper is organized as follows. In Section 2, the stability and convergence of the proposed network are discussed. Two illustrative examples and two applications are provided in Section 3. Finally some concluding remarks are drawn in Section 4.

\section{Stability}

In this section, we shall prove strictly the theoretical results for (3)-(4) appeared in Ref. 15. Two lemmas in Ref. 15 below pave a way for us to analyze the dynamics of (3)-(4).

Lemma 1. $(x, y) \in \mathscr{S}^{*}$ if and only if $x=(x-y)^{+}$ and $A x-B y=q$.

Lemma 2. Let

$$
\left\{\begin{array}{l}
\bar{x}=W^{-1}(B D z+q) \\
\bar{y}=W^{-1}(A z-q)
\end{array}\right.
$$


where $z \in R^{n}$. Then both (i) and (ii) below are true.

(i) $A \bar{x}-B D \bar{y}=q$ and $\bar{x}+\bar{y}=z$;

(ii) $(\bar{x}, D \bar{y}) \in \mathscr{S}^{*}$ if and only if $\bar{x}=(\bar{x}-\bar{y})^{+}$.

Proof. (i) Since $W=A+B D, \bar{x}+\bar{y}=z$ by (5) and

$$
\begin{gathered}
A W^{-1} B D=A W^{-1}(W-A)=A-A W^{-1} A \\
\quad=(W-A) W^{-1} A=B D W^{-1} A .
\end{gathered}
$$

Then $A \bar{x}-B D \bar{y}=A W^{-1}(B D z+q)-B D W^{-1}(A z-$ $q)=q$.

(ii) From (i), $A \bar{x}-B D \bar{y}=q$. Then $(\bar{x}, D \bar{y}) \in \mathscr{S}^{*}$ if and only if $\bar{x}=(\bar{x}-D \bar{y})^{+}$from Lemma 1 . Since $D$ is a positive diagonal matrix, we have

$$
\begin{aligned}
& \bar{x}=(\bar{x}-D \bar{y})^{+} \\
\Longleftrightarrow & \bar{x} \geqslant 0, D \bar{y} \geqslant 0, \bar{x}^{T} D \bar{y}=0 \\
\Longleftrightarrow \quad & \bar{x} \geqslant 0, \bar{y} \geqslant 0, \bar{x}^{T} \bar{y}=0 \\
\Longleftrightarrow & \bar{x}=(\bar{x}-\bar{y})^{+} .
\end{aligned}
$$

This completes the proof.

Lemma 3 in Ref. 15 below summarizes the relationship between the solution of problem (1) and the equilibrium point of (3).

Lemma 3. Let $\bar{x}$ and $\bar{y}$ be defined in (5), $\Omega^{*}=\{z \in$ $R^{n} \mid z$ solves $\left.\bar{x}=(\bar{x}-\bar{y})^{+}\right\}$, then the following are true.

(i) $z \in \Omega^{*}$ if and only if $(\bar{x}, D \bar{y}) \in \mathscr{S}^{*}$;

(ii) If $(x, y) \in \mathscr{S}^{*}$, then $z=x+D^{-1} y \in \Omega^{*}$;

(iii) There exists a bijective mapping between $\mathscr{S}^{*}$ and $\Omega^{*}$.

Proof. (i) can be obtained by the proof of Lemma 2(ii).

(ii) Since $(x, y) \in \mathscr{S}^{*}$ and $W=A+B D, B D z=$ $B D\left(x+D^{-1} y\right)=(W-A) x+B y=W x-q$. Then $x=W^{-1}(B D z+q)=\bar{x}$ by (5). Similarly, we have $y=D W^{-1}(A z-q)=D \bar{y}$. Thus $(\bar{x}, D \bar{y}) \in \mathscr{S}^{*}$ since $(x, y) \in \mathscr{S}^{*}$. Therefore $z=x+D^{-1} y \in \Omega^{*}$ by (i).

(iii) Consider the mapping $\varphi: \mathscr{S}^{*} \longmapsto \Omega^{*}$ defined by $\varphi(x, y)=x+D^{-1} y$ for each $(x, y) \in \mathscr{S}^{*}$, then $\varphi$ is well defined by (ii) and is surjective by (i). If $\varphi(x, y)=\varphi\left(x^{\prime}, y^{\prime}\right)$ for some $(x, y)$ and $\left(x^{\prime}, y^{\prime}\right) \in$ $\mathscr{S}^{*}$, then $z=x+D^{-1} y=x^{\prime}+D^{-1} y^{\prime} \in \Omega^{*}$ by (ii).
From the proof of (ii), we know that $x=W^{-1}(B D z+$ $q)=x^{\prime}$ and $y=D W^{-1}(A z-q)=y^{\prime}$. Thus $\varphi$ is injective.

Remark 1. Obviously $\bar{x}=(\bar{x}-\bar{y})^{+}$if and only if $\bar{y}=(\bar{y}-\bar{x})^{+}$. Then

$$
\begin{aligned}
& \bar{y}-(\bar{y}-\bar{x})^{+}=\bar{y}-(2 \bar{y}-z)^{+} \\
& \quad=W^{-1}(A z-q)-\left[2 W^{-1}(A z-q)-z\right]^{+}
\end{aligned}
$$

from (5). Thus an equivalent form of (3)-(4) can be written as:

- state equation

$$
\frac{d z}{d t}=-\rho\left[y-(2 y-z)^{+}\right],
$$

- output equation

$$
\left\{\begin{array}{l}
y=\widehat{R} z-s \\
x=z-D^{-1} y
\end{array}\right.
$$

where $\rho>0$ is a scaling constant, $\widehat{R}=W^{-1} A$ and $s=W^{-1} q$. This provides the compact form for LCP in (2) (See Example 3 in Section 3).

Now, we prove the following stability results of neural network (3)-(4) in Ref. 15.

Theorem 4. If there exists a positive diagonal matrix $\Lambda=\operatorname{diag}\left(\lambda_{1}, \lambda_{2}, \cdots, \lambda_{n}\right)$ such that the matrix $R^{T} \Lambda W^{-1} A$ is positive semi-definite, then neural network (3)-(4) is stable in the sense of Lyapunov, and the state trajectory $z(t)$ and the output trajectory $(x(t), y(t))$ of (3)-(4) with any initial point $z(0)=z^{0}$ will converge to a point in $\Omega^{*}$ and $\mathscr{S}^{*}$, respectively. In particular, neural network (3)-(4) is globally asymptotically stable when $\mathscr{S}^{*}=\left\{\left(x^{*}, y^{*}\right)\right\}$.

Proof. It is easy to see that $F(z)=x-(2 x-z)^{+}=$ $R z+s-[2(R z+s)-z]^{+}$is Lipschitz continuous in $R^{n}$. Then system (3) has a unique and continuous solution $z(t)$ with any initial point $z(0)=z^{0}$ for all $t \geqslant 0$.

Since $\lambda_{i}\left(w_{i}-w_{i}^{+}\right)\left(w_{i}^{+}-v_{i}\right)=\lambda_{i}\left(-w_{i}\right)^{+} v_{i}$ for all $i=1,2, \cdots, n, w \in R^{n}$ and $v \in R_{+}^{n}$, we have

$$
\left(w-w^{+}\right)^{T} \Lambda\left(w^{+}-v\right) \geqslant 0, \quad \forall w \in R^{n}, v \in R_{+}^{n} .
$$

Let $z^{*} \in \Omega^{*}$, then $R z^{*}+s \in R_{+}^{n}$ by (3), and

$$
[F(z)+R z+s-z]^{T} \Lambda\left[R\left(z-z^{*}\right)-F(z)\right] \geqslant 0
$$


by substituting $w=2(R z+s)-z$ and $v=R z^{*}+s$ into (8). Similarly, we have

$$
\left(R z^{*}+s-z^{*}\right)^{T} \Lambda\left[F(z)-R\left(z-z^{*}\right)\right] \geqslant 0
$$

from substituting $w=2\left(R z^{*}+s\right)-z^{*}$ and $v=$ $[2(R z+s)-z]^{+}$into (8). It follows from the above inequalities that

$$
\left[F(z)+\left(R-I_{n}\right)\left(z-z^{*}\right)\right]^{T} \Lambda\left[R\left(z-z^{*}\right)-F(z)\right] \geqslant 0 .
$$

Thus

$$
\begin{aligned}
& \left(z-z^{*}\right)^{T} \Lambda F(z) \geqslant\left(z-z^{*}\right)^{T}\left(I_{n}-R^{T}\right) \Lambda R\left(z-z^{*}\right) \\
& \quad+F(z)^{T} \Lambda F(z) \geqslant \lambda_{\text {min }}(\Lambda)\|F(z)\|^{2} \\
& \quad+\left(z-z^{*}\right)^{T} R^{T} \Lambda W^{-1} A\left(z-z^{*}\right)
\end{aligned}
$$

from $I_{n}-R=W^{-1}(W-B D)=W^{-1} A$.

Now, consider the function

$$
V(z)=\left(z-z^{*}\right)^{T} \Lambda\left(z-z^{*}\right),
$$

then

$$
\lambda_{\text {min }}(\Lambda)\left\|z-z^{*}\right\|^{2} \leqslant V(z) \leqslant \lambda_{\max }(\Lambda)\left\|z-z^{*}\right\|^{2}
$$

for all $z \in R^{n}$, and

$$
\begin{aligned}
& \frac{d}{d t} V[z(t)]=-2 \rho\left(z-z^{*}\right)^{T} \Lambda F[z(t)] \\
& \quad \leqslant-2 \rho \lambda_{\min }(\Lambda)\|F[z(t)]\|^{2}, \quad \forall t \geqslant 0
\end{aligned}
$$

from (9) and the positive semi-definiteness of $R^{T} \Lambda W^{-1} A$. Thus system (3)-(4) is Lyapunov stable. From (10)-(12) and following the similar argument as Refs. 17 and 20, we can prove that $z(t)$ converges to a point in $\Omega^{*}$.

From (4) and the analysis above, we see that the output trajectory $(x(t), y(t))$ also converges to a point in $\mathscr{S}^{*}$.

In particular, if $\mathscr{S}^{*}=\left\{\left(x^{*}, y^{*}\right)\right\}$, then $\Omega^{*}=\left\{z^{*}\right\}$ from Lemma 3 (iii), and the solution $z(t)$ of (3) will approach to $z^{*}$ by the analysis above. Thus neural network (3)-(4) is globally asymptotically stable.

Theorem 5. If there exists a positive diagonal matrix $\Lambda=\operatorname{diag}\left(\lambda_{1}, \lambda_{2}, \cdots, \lambda_{n}\right)$ such that the matrix $R^{T} \Lambda W^{-1} A$ is positive definite, then neural network (3)-(4) is globally exponentially stable.
Proof. From the proof of Theorem $4, \forall z^{0} \in R^{n}$, let $z(t)$ be the unique and continuous solution of (3) with $z(0)=z^{0}$ for all $t \geqslant 0$.

Since $S=R^{T} \Lambda W^{-1} A$ is positive definite, $\lambda_{\min }\left(S^{T}+S\right)>0, \Omega^{*}=\left\{z^{*}\right\}$ and

$$
\begin{aligned}
& \frac{d}{d t} V[z(t)] \leqslant-2 \rho\left[z(t)-z^{*}\right]^{T} S\left[z(t)-z^{*}\right] \\
& \quad \leqslant-\rho \lambda_{\min }\left(S^{T}+S\right)\left\|z(t)-z^{*}\right\|^{2} \\
& \quad \leqslant-\mu V[z(t)], \quad \forall t \geqslant 0
\end{aligned}
$$

from (9) and the second inequality of (11), where $\mu=\rho \lambda_{\min }\left(S^{T}+S\right) / \lambda_{\max }(\Lambda)$. Thus

$$
V[z(t)] \leqslant V\left(z^{0}\right) e^{-\mu t}, \quad \forall t \geqslant 0 .
$$

This and (11) imply that

$$
\left\|z(t)-z^{*}\right\| \leqslant \sqrt{\frac{\lambda_{\max }(\Lambda)}{\lambda_{\min }(\Lambda)}}\left\|z^{0}-z^{*}\right\| e^{-\mu t / 2}
$$

for all $t \geqslant 0$. This completes the proof.

Corollary 6 below shows the positive semidefiniteness of $R^{T} \Lambda W^{-1} A$ in Theorem 4 can be replaced with that of $A \Lambda^{-1} D B^{T}$ when $A$ or $B$ is nonsingular.

Corollary 6. Let $\Lambda=\operatorname{diag}\left(\lambda_{1}, \lambda_{2}, \cdots, \lambda_{n}\right)$ be a positive diagonal matrix. If $A$ or $B$ is nonsingular, then $R^{T} \Lambda W^{-1} A$ is positive semi-definite (positive definite) if and only if $A \Lambda^{-1} D B^{T}$ is positive semi-definite (positive definite).

Proof. If $A$ is nonsingular, so does $A^{-T} \Lambda W^{-1} A$. For each $z \in R^{n}$, let $v=A^{-T} \Lambda W^{-1} A z$, then $z=$ $A^{-1} W \Lambda^{-1} A^{T} v$ and

$$
\begin{aligned}
z^{T} R^{T} & \Lambda W^{-1} A z=z^{T}\left(I_{n}-W^{-1} A\right)^{T} \Lambda W^{-1} A z \\
& =v^{T} A \Lambda^{-1} W^{T} A^{-T}\left(I_{n}-A^{T} W^{-T}\right) A^{T} v \\
& =v^{T} A \Lambda^{-1}\left(W^{T}-A^{T}\right) v=v^{T} A \Lambda^{-1} D B^{T} v
\end{aligned}
$$

from $R=W^{-1} B D=I_{n}-W^{-1} A$ since $W=A+B D$. Thus the results holds by the non-singularity of $A^{-T} \Lambda W^{-1} A$.

If $B$ is nonsingular, so does $B^{-T} D^{-1} \Lambda R$. Similar to the analysis above, the results can be obtained by

$$
z^{T} R^{T} \Lambda W^{-1} A z=v^{T} A \Lambda^{-1} D B^{T} v, \quad \forall z \in R^{n},
$$

where $v=B^{-T} D^{-1} \Lambda R z$. 
Obviously, both $A$ and $B$ are nonsingular when $R^{T} \Lambda W^{-1} A$ or $A \Lambda^{-1} D B^{T}$ is positive definite. Then the positive definiteness of $R^{T} \Lambda W^{-1} A$ in Theorem 5 can be replaced with that of $A \Lambda^{-1} D B^{T}$ from Corollary 6.

Corollary 7 in Ref. 15 below shows that (3)-(4) is asymptotically stable and has no any parameter when problem (1) is monotone.

Corollary 7. If problem (1) is monotone, i.e., for each pair of points $x, y \in R^{n}$,

$$
A x-B y=0 \Rightarrow x^{T} y \geqslant 0,
$$

then neural network (3)-(4) with $D=I_{n}$ is stable in the sense of Lyapunov, and the state trajectory $z(t)$ and the output trajectory $(x(t), y(t))$ of (3)-(4) with any initial point $z(0)=z^{0}$ will converge to a point in $\Omega^{*}$ and $\mathscr{S}^{*}$, respectively.

Proof. From (13), it is easy to verify that $A+B$ is nonsingular. $\forall z, z^{\prime} \in R^{n}$, let $\bar{x}$ and $\bar{y}$ defined in (5) with $D=I_{n}, \bar{x}^{\prime}=W^{-1}\left(B z^{\prime}+q\right)$ and $\bar{y}^{\prime}=W^{-1}\left(A z^{\prime}-\right.$ $q$ ), where $W=A+B$, then

$$
A \bar{x}-B \bar{y}=q \quad \text { and } \quad A \bar{x}^{\prime}-B \bar{y}^{\prime}=q
$$

by Lemma 2(i). Thus $A\left(\bar{x}-\bar{x}^{\prime}\right)-B\left(\bar{y}^{\prime}-\bar{y}^{\prime}\right)=0$ and

$$
\left(\bar{x}-\bar{x}^{\prime}\right)^{T}\left(\bar{y}-\bar{y}^{\prime}\right)=\left(z-z^{\prime}\right)^{T} R^{T} W^{-1} A\left(z-z^{\prime}\right) \geqslant 0
$$

for all $z, z^{\prime} \in R^{n}$ from (13) and $R=W^{-1} B$. Thus $R^{T} W^{-1} A$ is positive semi-definite and the result holds from Theorem 4 with $\Lambda=I_{n}$.

From Corollary 6 and the proof of Corollary 7 , we have following remark to judge that problem (1) is nonmonotone.

Remark 2. Problem (1) is nonmonotone if one of the following conditions is satisfied:

(i) $A+B$ is singular;

(ii) $A^{T}(A+B)^{-T}(A+B)^{-1} B$ is indefinite;

(iii) $A$ or $B$ is nonsingular and $A B^{T}$ is indefinite.

Moreover, we have the following remark on the choice of matrices $\Lambda$ and $D$ for (3)-(4) from the analysis above.

Remark 3. (i) If $A+B$ is nonsingular, then one can set $D=I_{n}$ in (3)-(4), only need to choose one matrix $\Lambda$ and check the positive semi-definiteness of
$B^{T} W^{-T} \Lambda W^{-1} A$ or $A \Lambda^{-1} B^{T}$ for Theorem 4 and the positive definiteness of $A \Lambda^{-1} B^{T}$ for Theorem 5, respectively. In particular, (3)-(4) has no any parameter at this case.

(ii) If $A+B$ is singular, then one can set $\Lambda=I_{n}$ in (3)-(4), only need to choose one matrix $D$ such that $W$ is nonsingular, and check the positive semidefiniteness of $R^{T} W^{-1} A$ or $A D B^{T}$ for Theorem 4 and the positive definiteness of $A D B^{T}$ for Theorem 5, respectively.

\section{Illustrative Examples}

In this section, four examples are provided to illustrate both the theoretical results in Section 2 and the simulation performance of the neural network (3)(4). The simulation is conducted in Matlab, and the ode solver used is ODE23s which is a stiff medium order method.

Example 1 below shows the simulation performance of neural network (3)-(4) for a nonmonotone problem with infinite number of solutions.

Example 1. Consider problem (1), where

$$
\begin{gathered}
A=\left(\begin{array}{ccc}
1 / 2 & 1 / 4 & 0 \\
-1 / 2 & 1 / 2 & -1 / 3 \\
0 & 0 & 1 / 3
\end{array}\right), \\
B=\left(\begin{array}{ccc}
1 & 0 & 0 \\
0 & 1 & -1 / 2 \\
-1 & -1 & 1
\end{array}\right)
\end{gathered}
$$

and $q=(1,-2,1)^{T}$. Then it has infinite number of solutions $x^{*}=\left(2,0,3\left(1-y_{2}^{*}\right)\right)^{T}$ and $y^{*}=$ $\left(0, y_{2}^{*}, 0\right)^{T}\left(0 \leqslant y_{2}^{*} \leqslant 1\right)$.

Although both $A$ and $B$ are nonsingular, this problem is nonmonotone from Remark 2(iii) since $A B^{T}$ is indefinite. Moreover, one can verify that $W=A+B$ is nonsingular and $A \Lambda^{-1} B^{T}$ with $\Lambda=$ $\operatorname{diag}\{1,1 / 2,1 / 9\}$ is positive semi-definite. Then Theorem 4 ensures the stability and convergence of neural network (3)-(4) with $D=I_{3}$ for this problem from Corollary 6 and Remark 3(i). When applied to it, all simulation results show that the state trajectory $z(t)$ and the output trajectory $(x(t), y(t))$ of (3)-(4) always converge to one of its equilibrium point $z^{*}=\left(2, z_{2}^{*}, 3\left(1-z_{2}^{*}\right)\right)^{T}\left(0 \leqslant z_{2}^{*} \leqslant 1\right)$ and 
a solution of this problem respectively, where $x^{*}=$ $\left(2,0,3\left(1-z_{2}^{*}\right)\right)^{T}$ and $y^{*}=\left(0, z_{2}^{*}, 0\right)^{T}$. For example, let $\rho=10$ and $z^{0}=(5,-5,0)^{T}$, we get an equilibrium point $z^{*}=(2,0.0644607,2.806618)^{T}$ of (3) and a solution $\left(x^{*}, y^{*}\right)$ of this problem, where $x^{*}=(2,0,2.806618)^{T}$ and $y^{*}=(0,0.0644607,0)^{T}$.

Fig. 1 depicts the convergence behavior of the error $\left\|x(t)-x^{*}\right\|+\left\|y(t)-y^{*}\right\|$ based on (3)-(4) with 30 random initial points and $\rho=10$.

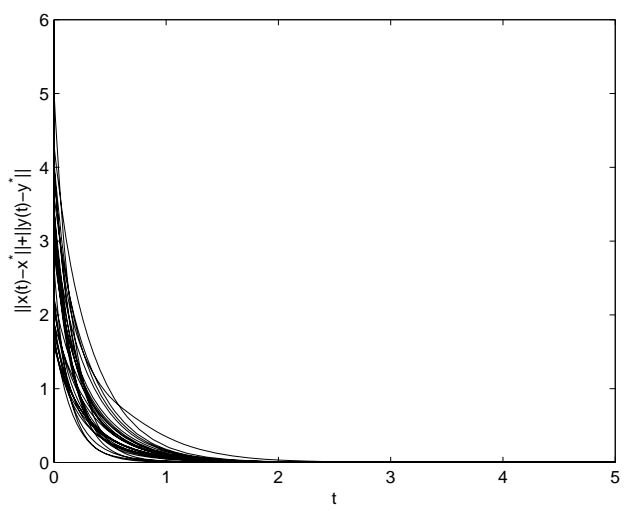

Fig. 1. Convergence behavior of the error $\left\|x(t)-x^{*}\right\|+$ $\left\|y(t)-y^{*}\right\|$ based on (3)-(4) with 30 random initial points in Example 1

As Example 2 in Ref. 15, Example 1 can be transformed into LCP $\left(A^{-1} B, A^{-1} q\right.$ ) (or LCP $\left.\left(B^{-1} A,-B^{-1} q\right)\right)$ in (2), and the simulation results show that the existing neural networks in Refs. 14 and 18-22 for these resulting LCPs are stable. However, unlike model (3)-(4), their stability and convergence cannot be guaranteed since both $A^{-1} B$ and $B^{-1} A$ are asymmetric and indefinite.

Example 2 below shows the simulation performance of neural network (3)-(4) for a monotone problem.

Example 2. Consider problem (1), where

$$
\begin{aligned}
A & =\left(\begin{array}{cccc}
1 & -1 & 0 & 0 \\
-1 & 1 & 0 & 0 \\
0 & 0 & 0 & -1 \\
0 & 0 & -1 & 0
\end{array}\right), \\
B & =\left(\begin{array}{cccc}
0 & -1 & 0 & 0 \\
-1 & 0 & 0 & 0 \\
0 & 0 & 1 & -1 \\
0 & 0 & -1 & 1
\end{array}\right)
\end{aligned}
$$

and $q=(0,0,0,0)^{T}$. Then it has infinite number of solutions $x^{*}=\left(x_{1}^{*}, x_{1}^{*}, 0,0\right)^{T}$ and $y^{*}=\left(0,0, y_{3}^{*}, y_{3}^{*}\right)^{T}$ $\left(x_{1}^{*} \geqslant 0, y_{3}^{*} \geqslant 0\right)$.

Even though both $A$ and $B$ are singular, one can verify that this problem is monotone. Then it can be solved by neural network (3)-(4) with $D=I_{4}$ from Corollary 7. When applied to this problem, all simulation results show that the state trajectory $z(t)$ and the output trajectory $(x(t), y(t))$ of (3)-(4) with $D=I_{4}$ always converge to one of its equilibrium points $z^{*}=\left(z_{1}^{*}, z_{1}^{*}, z_{3}^{*}, z_{3}^{*}\right)^{T}\left(z_{1}^{*} \geqslant 0, z_{3}^{*} \geqslant 0\right)$ and a solution $\left(x^{*}, y^{*}\right)$ of this problem respectively, where $x^{*}=\left(z_{1}^{*}, z_{1}^{*}, 0,0\right)^{T}$ and $y^{*}=\left(0,0, z_{3}^{*}, z_{3}^{*}\right)^{T}$. For example, let $\rho=10$ and $z^{0}=(3,2.5,1,0.6)^{T}$, we get an equilibrium point $z^{*}=(2.75,2.75,0.8,0.8)^{T}$ of (3) and a solution $\left(x^{*}, y^{*}\right)$ of this problem with $x^{*}=$ $(2.75,2.75,0,0)^{T}$ and $y^{*}=(0,0,0.8,0.8)^{T}$. Fig. 2 depicts the convergence behavior of the error $\| z(t)-$ $z^{*} \|$ based on (3)-(4) with 30 random initial points and $\rho=10$.

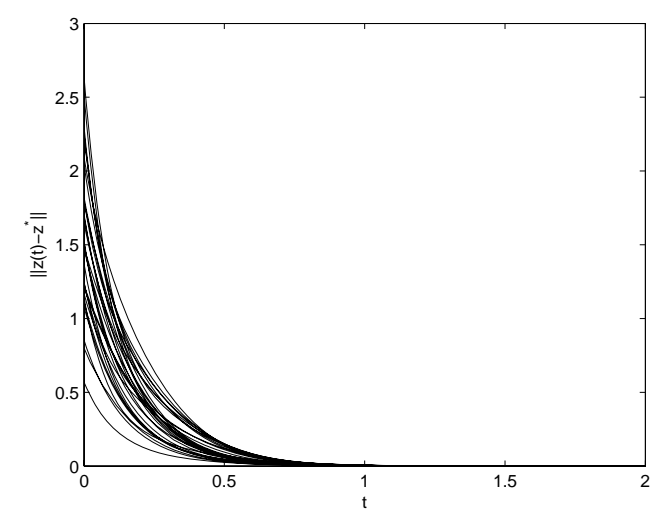

Fig. 2. Convergence behavior of the error $\left\|z(t)-z^{*}\right\|$ based on (3)-(4) with 30 random initial points in Example 2

Example 3. Consider a transportation problem ${ }^{8}$ :

$$
\left\{\begin{aligned}
\min & \sum_{i=1}^{m} \sum_{i=1}^{n} c_{i j} u_{i j}, \\
\text { s.t. } & \sum_{j=1}^{n} u_{i j} \leqslant a_{i}, \quad i=1,2, \cdots, m, \\
& \sum_{i=1}^{m} u_{i j} \geqslant b_{j}, \quad j=1,2, \cdots, n, \\
& u_{i j} \geqslant 0 \quad i=1,2, \cdots, m, \quad j=1,2, \cdots, n .
\end{aligned}\right.
$$

Then it can be written as

$$
\left\{\begin{array}{cl}
\min & c^{T} u \\
\text { s.t. } & G u \geqslant d, \quad u \geqslant 0,
\end{array}\right.
$$


where $u=\left(u_{11}, u_{12}, \cdots, u_{1 n}, \cdots, u_{m 1}, u_{m 2}, \cdots, u_{m n}\right)^{T}$, $c=\left(c_{11}, c_{12}, \cdots, c_{1 n}, \cdots, c_{m 1}, c_{m 2}, \cdots, c_{m n}\right)^{T} \in R^{m n}$, $d=\left(-a_{1},-a_{2}, \cdots,-a_{m}, b_{1}, b_{2}, \cdots, b_{n}\right)^{T}$ and $G=$ $\left(-G_{1}^{T}, G_{2}^{T}\right)^{T}$ with $G_{2}=\left(I_{n}, I_{n}, \cdots, I_{n}\right)_{n \times m n}$,

$$
G_{1}=\left(\begin{array}{cccc}
g_{n}^{T} & 0 & \cdots & 0 \\
0 & g_{n}^{T} & \cdots & 0 \\
\vdots & \vdots & \ddots & \vdots \\
0 & 0 & \cdots & g_{n}^{T}
\end{array}\right)_{m \times m n},
$$

$g_{n}=(1,1, \cdots, 1)^{T}$ and $0=(0,0, \cdots, 0) \in R^{n}$.

By Kuhn-Tucker Theorem ${ }^{9}, u^{*}$ is an optimal solution of (14) if and only if there exists a $\lambda^{*} \in R^{m+n}$ such that $y^{*}=\left(u^{* T}, \lambda^{* T}\right)^{T}$ is a solution of LCP $(M, q)$ in (2) with $q=\left(c^{T},-d^{T}\right)^{T}$,

$$
M=\left(\begin{array}{cc}
O & -G^{T} \\
G & O
\end{array}\right)
$$

and $O$ being the zero matrix of proper order. Then from Remark 1 and Corollary 6, neural network (3)(4) for (2) can be simplified as:

- state equation

$$
\frac{d z}{d t}=-\rho\left[y-(2 y-z)^{+}\right]
$$

- output equation

$$
y=R(z-q),
$$

where $R=\left(I_{n}+M\right)^{-1}$ and $\rho>0$ is a scaling constant. Obviously this model has a one-layer structure, while existing models in Refs. 16-18 are twolayer structure. Furthermore, we have the following result for (16)-(17) from Theorems 4-5, which shows that (16)-(17) can be used to solve some nonmonotone LCP (see Example 1 in Ref. 15).

Corollary 8. If there exists a positive diagonal matrix $\Lambda=\operatorname{diag}\left(\lambda_{1}, \lambda_{2}, \cdots, \lambda_{n}\right)$ such that $\Lambda M$ is positive semi-definite, then neural network (16)-(17) is stable in the sense of Lyapunov, and the state trajectory $z(t)$ and the output trajectory $y(t)$ of (16)-(17) with any initial point $z(0)=z^{0}$ will converge to a point in $\Omega^{*}$ and a solution of problem (2), respectively. Furthermore, (16)-(17) is globally exponentially stable when $\Lambda M$ is positive definite.
Proof. Since $\Lambda M$ is positive semi-definite, $\Lambda\left(I_{n}+\right.$ $M)$ is positive definite. Then $\Lambda\left(I_{n}+M\right)$ is nonsingular, and so does $I_{n}+M$. Thus the result can be obtained from Theorems 4-5 and Corollary 6.

In particular, since $M$ defined in (15) is positive semi-definite, Corollary 8 ensures the stability and convergence of (16)-(17) when applied to problem (14). At this case,

$$
R=\left(\begin{array}{cc}
I_{m n}-G^{T} H G & G^{T} H \\
-H G & H
\end{array}\right),
$$

where

$$
\begin{aligned}
H= & \left(I_{m+n}+D D^{T}\right)^{-1}=\left(\begin{array}{cc}
\frac{1}{n+1} I_{m} & O \\
O & \frac{1}{m+1} I_{n}
\end{array}\right) \\
& +\frac{1}{m+n+1}\left(\begin{array}{cc}
\frac{n}{n+1} g_{m} g_{m}^{T} & g_{m} g_{n}^{T} \\
g_{n} g_{m}^{T} & \frac{m}{m+1} g_{n} g_{n}^{T}
\end{array}\right) .
\end{aligned}
$$

For example, let $m=n=4, c=(3,2,4,1,2,1.5$, $2.5,3,4,3.5,3,1.5,3,2,1,2)^{T}, a_{i}=2$ and $b_{i}=1.5$ for $i=1,2 \cdots n$, then (14) has a unique solution $u^{*}=$ $(0,0.5,0,1.5,1.5,0.5,0,0,0,0,0,0,0,0.5,1.5,0)^{T}$.

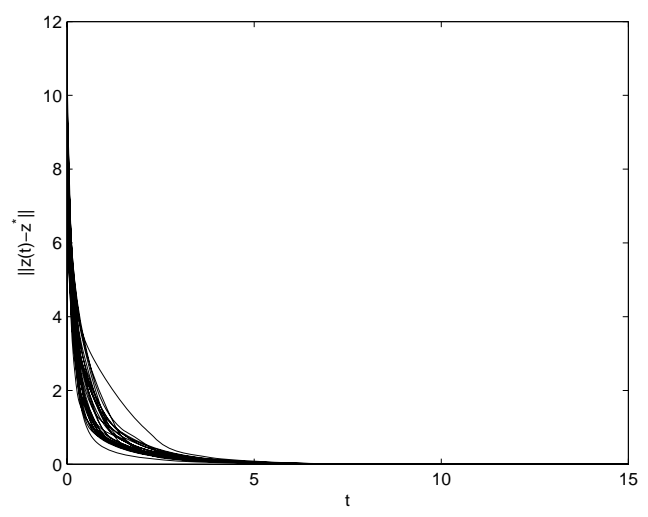

Fig. 3. Convergence behavior of error $\left\|z(t)-z^{*}\right\|$ based on (16)-(17) with 30 random initial points in Example 3.

When applied to this problem, all simulation results show that the state variables $z(t)$ and the output variables $y(t)$ of (16)-(17) always converge to one of its equilibrium points $z^{*}=$ $\left(0.5,0.5,3,1.5,1.5,0.5,2,2.5,1+z_{12}^{*}, 1+z_{12}^{*}, 1.5+\right.$ $z_{12}^{*}, z_{12}^{*}, 0.5,0.5,1.5,1, z_{17}^{*}, 0.5+z_{17}^{*}, 2, z_{17}^{*}, 2.5+z_{17}^{*}$, $\left.2+z_{17}^{*}, 1+z_{17}^{*}, 1+z_{17}^{*}\right)^{T}\left(0 \leqslant z_{12}^{*} \leqslant 0.5,0 \leqslant z_{17}^{*} \leqslant\right.$ $0.5)$ and $y^{*}=\left(u^{*}, \lambda^{*}\right)$ respectively, where $\lambda^{*}=$ $\left(z_{17}^{*}, 0.5+z_{17}^{*}, 0, z_{17}^{*}, 2.5+z_{17}^{*}, 2+z_{17}^{*}, 1+z_{17}^{*}, 1+\right.$ 
$\left.z_{17}^{*}\right)^{T}$. Thus we obtain the solution $u^{*}$ of (14). For example, Fig. 3 depicts the convergence behavior of the error $\left\|z(t)-z^{*}\right\|$ based on (16)-(17) with 30 random initial points and $\rho=10$.

Example 4. Consider a class of the absolute equations ${ }^{6}$ :

$$
|u|=N u-q,
$$

where $|u|=\left(\left|u_{1}\right|,\left|u_{2}\right|, \cdots,\left|u_{n}\right|\right)^{T}, N \in R^{n \times n}$ and $q \in$ $R^{n}$.

Let $x=\max \{u, 0\}$ and $y=\max \{-u, 0\}$, then $u=x-y,|u|=x+y$ and (18) becomes (1) with $A=N-I_{n}$ and $B=I+N_{n}$. When $N$ is nonsingular, neural network (3)-(4) for (18) can be simplified as:

- state equation

$$
\frac{d z}{d t}=\rho\left[u^{+}-\frac{1}{2}(z+u)\right]=\frac{\rho}{2}(|u|-z),
$$

- output equation

$$
u=R(z+q),
$$

where $R=N^{-1}$ and $\rho>0$ is a scaling constant.

Since $\left(N-I_{n}\right)^{T} N^{-T} N^{-1}\left(N+I_{n}\right)=N^{-T}(N-$ $\left.I_{n}\right)^{T}\left(N+I_{n}\right) N^{-1}$, the positive semi-definiteness (definiteness) of $\left(N-I_{n}\right)^{T} N^{-T} N^{-1}\left(N+I_{n}\right)$ is equivalent to that of $N^{T} N-I_{n}$. Then we have the following result for (19)-(20) from Theorems 4-5.

Corollary 9. If $\lambda_{\min }\left(N^{T} N\right) \geqslant 1$, then neural network (19)-(20) is stable in the sense of Lyapunov, and for any $z^{0} \in R^{n}$, the state trajectory $z(t)$ and the output trajectory $u(t)$ of (19)-(20) with initial point $z(0)=z^{0}$ will converge to one of its equilibrium point and a solution of problem (18), respectively. Furthermore, (19)-(20) is globally exponentially stable when $\lambda_{\min }\left(N^{T} N\right)>1$.

As an example, let $q=(4,3,2,3)^{T}$ and

$$
N=\left(\begin{array}{cccc}
4 & -2 & 3 & -1 \\
-2 & 3 & 2.5 & -1.5 \\
1.5 & 2.5 & 4 & 0.5 \\
2 & -1 & 2.5 & 3
\end{array}\right)
$$

then (18) has a unique solution $u^{*}=(-0.809917$, $-0.975207,1.933884,-0.297521)^{T}$.
It is easy to verify that $N$ is indefinite and $\lambda_{\min }\left(N^{T} N\right)=1.00052$. Then Corollary 9 ensures that neural network (19)-(20) is globally exponentially stable for this problem. When applied to it, all simulation results show that the state variables $z(t)$ and the output variables $u(t)$ of (19)-(20) are exponentially stable at $z^{*}=\left|u^{*}\right|$ and $u^{*}$, respectively. For example, Fig. 4 depicts the output trajectories $u(t)$ of (19)-(20) with 30 random initial points and $\rho=10$ converging to $u^{*}$.

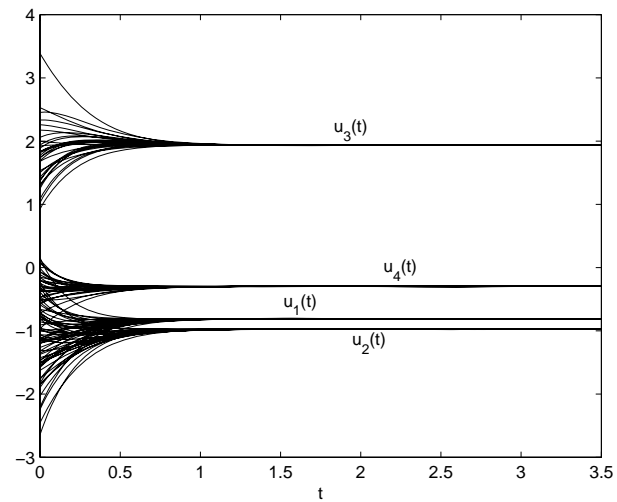

Fig. 4. Transient behavior of the output trajectories of (19)(20) with 30 random initial points in Example 4.

\section{Conclusion}

In this paper, we strictly prove the stability and convergence of a one-layer neural network proposed by Gao and Wang ${ }^{15}$, which is designed to solve a class of horizontal linear complementarity problem (HLCP). Two applications and two numerical examples are provided to show further the validity and performance of this system.

\section{Acknowledgments}

The authors would like to thank Prof. Yuping Wang and Prof. Ping Guo for many helpful discussions. The authors are also very grateful to the Associate Editor and anonymous reviewers for their comments and constructive suggestions on an earlier version of this paper. 


\section{References}

1. M. S. Gowda, "On the extended linear complementarity problem," Mathematical Programming, 72, 33-50 (1996).

2. O. L. Mangasarian and J. S. Pang, "The extended linear complementarity problem," SIAM Journal on Matrix Analysis and Applications, 16, 359-368 (1995).

3. M. S. Gowda and R. Sznajder, "The generalized order linear complementarity problems," SIAM J. Matrix Anal. Appl., 15, 79-795 (1994).

4. S. R. Mohan, S. K. Neogy and R. Sridhar, "The generalized linear complementarity problem revisited," Mathematical Programming, 74, 197-218 (1996).

5. M. V. Solodov, "Some optimization reformulations of the extended linear complementarity problem," Computational Optimization and Applications, 13, 187200 (1999).

6. O. L. Mangasarian and R. R. Meyer, "Absolute value equations," Linear Algebra and its Applications, 419, 359-367 (2006).

7. L. Fernandes, A. Friedlander, M. Guedes and J. Júdice, "Solution of a general linear complementarity problem using smooth optimization and its application to bilinear programming and LCP," Applied Mathematics and Optimization, 43, 1-19 (2001).

8. J. Noceda and S. J. Wright, Numerical Optimization, Springer, New York, 1999.

9. M. S. Bazaraa, H. D. Sherali and C. M. Shetty, Nonlinear programming-theory and algorithms, 2nd ed. New York: Wiley, 1993.

10. M. S. Gowda, "Reducing a monotone horizontal LCP to a LCP," Applied Mathematics Letter, 8, 97-100 (1995).

11. D. Ralph, "A stable homotopy approach to horizontal linear complementarity problems," Control and Cybernetics, 31, 575-600 (2002).

12. N. H. Xiu and J. Z. Zhang, "A smoothing GaussNewton method for the generalized HLCP", Jour- nal of Computational and Applied Mathematics, 129, 195-208 (2001).

13. Y. Zhang, "On the convergence of a class of infeasible interior-point algorithms for the horizontal linear complementarity problem", SIAM Journal on $\mathrm{Op}$ timization, 4, 208-227 (1994).

14. T. L. Friesz, D. H. Bernstein, N. J. Mehta, R. L. Tobin and S. Ganjlizadeh, "Day-to-day dynamic network disequilibria and idealized traveler information systems," Operations Research, 42, 1120-1136 (1994).

15. X. B. Gao and J. Wang, "A neural network for a class of horizontal linear complementarity problems," 2011 Seventh International Conference on Computational Intelligence and Security (CIS11), 353-357 (2011).

16. X. B. Gao, "A nerual network for a class of extended linear variational inequaties," Chinese JournL of Electronics, 10, 471-475 (2001).

17. X. B. Gao and L.-Z. Liao, "A novel neural network for a class of convex quadratic minimax problems," $\mathrm{Neu}$ ral Computation, 18, 1818-1846 (2006).

18. Y. S. Xia and J. Wang, "A general methodology for designing globally convergent optimization neural networks," IEEE Trans. on Neural Networks, 9, 13111343 (1998).

19. Y. S. Xia and J. Wang, "A general projection neural network for solving monotone variatonal inequalities and related optimization prpblems", IEEE Trans. on Neural Networks, 15, 318-328 (2004).

20. X. B. Gao, "Exponential stability of globally projected dynamic systems," IEEE Trans. on Neural Networks, 14, 426-431 (2003).

21. X. B. Gao, L.-Z. Liao and W. M. Xue, "A neural network for a class of convex quadratic minimax problems with constraints," IEEE Trans. on Neural Networks, 15, 622-628 (2004).

22. Y. S. Xia, "Further results on global convergence and stability of globally projected dynamic systems," Journal of Optimization Theory and Applications, 122, 627-649 (2004). 